ARTICLE

https://doi.org/10.1038/s41467-019-08505-6

\title{
Spontaneous formation and relaxation of spin domains in antiferromagnetic spin-1 condensates
}

\author{
K. Jiménez-García1,2, A. Invernizzi ${ }^{1,3}$, B. Evrard ${ }^{1}$, C. Frapolli1,3, J. Dalibard¹ \& F. Gerbier ${ }^{1}$
}

Many-body systems at low temperatures generally organize themselves into ordered phases, whose nature and symmetries are captured by an order parameter. This order parameter is spatially uniform in the simplest cases, for example the macroscopic magnetization of a ferromagnetic material. Non-uniform situations also exist in nature, for instance in antiferromagnetic materials, where the magnetization alternates in space, or in the so-called stripe phases emerging for itinerant electrons in strongly correlated materials. Understanding such inhomogeneously ordered states is of central importance in many-body physics. Here we study experimentally the magnetic ordering of itinerant spin-1 bosons in inhomegeneous spin domains at nano-Kelvin temperatures. We demonstrate that spin domains form spontaneously, that is purely because of the antiferromagnetic interactions between the atoms and in the absence of external magnetic forces, after a phase separation transition. Furthermore, we explore how the equilibrium domain configuration emerges from an initial state prepared far from equilibrium.

\footnotetext{
${ }^{1}$ Laboratoire Kastler Brossel, Collège de France, CNRS, ENS-PSL Research University, Sorbonne Université, 11 Place Marcelin Berthelot, 75005 Paris, France. ${ }^{2}$ Present address: Centro de Investigación y Estudios Avanzados del Instituto Politécnico Nacional-Unidad Querétaro, 76230 Querétaro, Mexico. ${ }^{3}$ Present address: Safran REOSC, Avenue de la tour Maury, 91280 Saint-Pierre-du-Perray, France. Correspondence and requests for materials should be addressed to F.G. (email: fabrice.gerbier@lkb.ens.fr)
} 
O uantum gases of ultracold atoms offer an unprecedented platform to study complex, multicomponent quantum fluids in- and out-of-equilibrium ${ }^{1-3}$. In quantum gases with several Zeeman components simultaneously confined in an optical dipole trap, Van der Waals ${ }^{4-10}$ or dipole-dipole ${ }^{11,12}$ interactions drive internal conversion between the Zeeman components. For bosonic atoms, this leads at very low temperatures to Bose-Einstein condensation in a superposition of the internal states where long-range phase coherence, superfluidity and magnetic ordering can all take place. For instance, Josephsonlike spin oscillations due to spin-changing interactions have been observed experimentally $^{7-9}$, and spin superfluidity demonstrated in recent experiments with sodium atoms ${ }^{13,14}$.

A major question that arises for multicomponent fluidsquantum or classical-is the stability of spatially homogeneous phases toward phase separation ${ }^{1}$. In cold atom experiments, phase separation has been observed in numerous multicomponent systems, either in dual species Bose-Bose or Bose-Fermi mixtures ${ }^{15-23}$ or for single-species quantum gases with several hyperfine components, for example two-component imbalanced Fermi gases with strong interactions ${ }^{24}$ or bosonic mixtures of hyperfine states ${ }^{25-28}$. Reaching equilibrium in dual species mixtures can be difficult if inelastic losses are strong, for instance near a Feshbach resonance. In that context, metastable phase-separated configurations were reported in ref. ${ }^{20}$. Furthermore, in many cases the different components experience different trapping potentials due to different magnetic moments or masses. A species- or spin-dependent trapping potential can strongly influence phase separation in a trapped system, to the point where it becomes the main factor deciding its occurrence instead of interatomic interactions ${ }^{1,29}$.

In this work, we study the formation of spin domains in a quasi-one-dimensional (1d) spinor Bose-Einstein condensate (BEC) in an external, spatially uniform magnetic field without any external magnetic force. The condensate is made from sodium atoms carrying a hyperfine spin $F=1$. The spindependent interactions have an antiferromagnetic character that leads to phase separation ${ }^{30-34}$. Early experiments observed spin domains in a $F=1$ sodium BEC immersed in a magnetic field gradient around $10 \mathrm{mG} / \mathrm{cm}^{30,32,33}$. The magnetic force produced by the gradient makes the $m_{F}= \pm 1$ Zeeman components migrate to opposite sides of the trap, with the $m_{F}=0$ component inbetween. Without applied gradient, only the miscible $m_{F}= \pm 1$ phase was observed in ref. ${ }^{30}$. References ${ }^{35-37}$ pointed out theoretically that $m_{F}=0$ spin domains should also form without any applied gradient. For a gas in a box, the domain should preferentially move to one side of the box to have only one interface, with $m_{F}=0$ on one side of the box and $m_{F}= \pm 1$ on the other. For a trapped gas, the gain in interaction energy when the $m_{F}=0$ domain is located in the center of the trap outweights the energetic cost of a second interface $30,31,37$.

Another important question besides the nature of the equilibrium state is whether this state can be reached on a timescale compatible with the lifetime of the atomic sample. References 32,33 studied relaxation in a strong applied magnetic field gradient, observing that metastable configurations can persist for seconds. Several experiments, mostly using $F=1{ }^{87} \mathrm{Rb}$ atoms with ferromagnetic interactions ${ }^{27,28,34,38-42}$, studied the dynamical formation of nonequilibrium spin domains after a quench. Reaching an equilibrium state appears difficult for ${ }^{87} \mathrm{Rb}$ atoms due to the weakness of spin interactions ${ }^{43}$. Other experiments with $F=$ 1 sodium atoms observed the formation of short-lived domains across a quantum phase transition and studied their equilibration dynamics ${ }^{44,45}$. However, heating due to the experimental arrangement prevented to study the long-time regime and the approach to the expected equilibrium state. The relation between the formation of spin domains after a quench and the Kibble-Zurek mechanism has also been discussed ${ }^{46}$.

The spin-1 quantum gas in our experiments is confined in a spin-independent and highly elongated trap, realizing an effectively $1 \mathrm{~d}$ spinor gas where phase separation occurs only along the weak axis. We take special care to compensate for magnetic field gradients along that axis (canceling them below the $\mathrm{mG} / \mathrm{cm}$ level) to ensure the domains form in a negligible magnetic force. We measure the equilibrium spatial distributions, which reflect (up to interface effects that we quantify) the phase boundaries for systems with homogeneous particle density. We find qualitative agreement but quantitative differences between the measured equilibrium distributions and $T=0$ mean-field theory. We attribute these differences to thermal fluctuations, which play an important role due to the low-energy scales associated with spin ordering, and the low dimensionality. In the second part of the article, we adress the issue of relaxation to equilibrium in a gradient-free situation. We prepare a spin configuration far from equilibrium and monitor how it relaxes to equilibrium. We observe a slow relaxation on a time scale of several seconds, and a spin dynamics that points to spin-mixing collisions as the underlying relaxation mechanism.

\section{Results}

Experimental system. Our experiments are performed with a gas of ${ }^{23} \mathrm{Na}$ atoms trapped in a spin-independent optical trap with frequencies $\omega_{x} \ll \omega_{y}=\omega_{z}$ (Fig. 1a). With a total atom number around $N \approx 10^{4}$, the chemical potential of a single-component gas at low temperatures is $\mu \sim 0.5 \hbar \omega_{y}$. This implies a quasi-1d regime where the transverse motion is almost frozen to the ground state of the harmonic potential. The total atom number decays with a measured 1/e lifetime around $50 \mathrm{~s}$, presumably limited by residual evaporation and three-body recombination.

We measure the linear integrated densities $\rho_{1 \mathrm{~d}, m_{F}}(x)=$ $\int d y d z \rho_{m_{F}}(\mathbf{r})$ along the weak axis of the trap after a short expansion in a magnetic field gradient that separates all three components $m_{F}=0, \pm 1$ by the Stern-Gerlach effect. Here and in the following, $\rho_{m_{F}}$ denotes the partial density of the Zeeman component with magnetic quantum number $m_{F}=0, \pm 1, \rho=$ $\sum_{m_{F}} \rho_{m_{F}}$ the total density, and the subscript " $1 d$ " always indicates linear quantities integrated over the transverse coordinates $y, z$.

Because of its quasi-1d character, the bosonic quantum gases studied in our experiment are quasi-condensates ${ }^{4-52}$ : density fluctuations are essentially frozen, as for a true condensate, but phase fluctuations along the weak axis of the trap can be substantial at finite temperatures. These phase fluctuations do not affect the thermodynamic properties of the mixture, but they show up as density stripes in time-of-flight images ${ }^{49,51,52}$. The density profiles reported in this article are averaged profiles over many (typically several tens) repetitions of the experiment to suppress the signature of phase fluctuations. Because of the very weak expansion along $x$, the observed average distributions reflect the linear in-trap density distributions to a good approximation. We also take special care to cancel residual magnetic forces that could affect the spatial distributions (see Methods). This is reflected in the nearly symmetric linear distributions of the spin components (Fig. 1b-d).

Brief review of ultracold spin-1 gases. Before discussing our results, we first review the salient features of $F=1$ spinor condensates $^{3}$. At very low temperatures, Bose-Einstein condensation leads to a macroscopic occupation of a single-particle state $\Psi$, a superposition of all three Zeeman states behaving as a threedimensional vector. The equilibrium many-body state is 

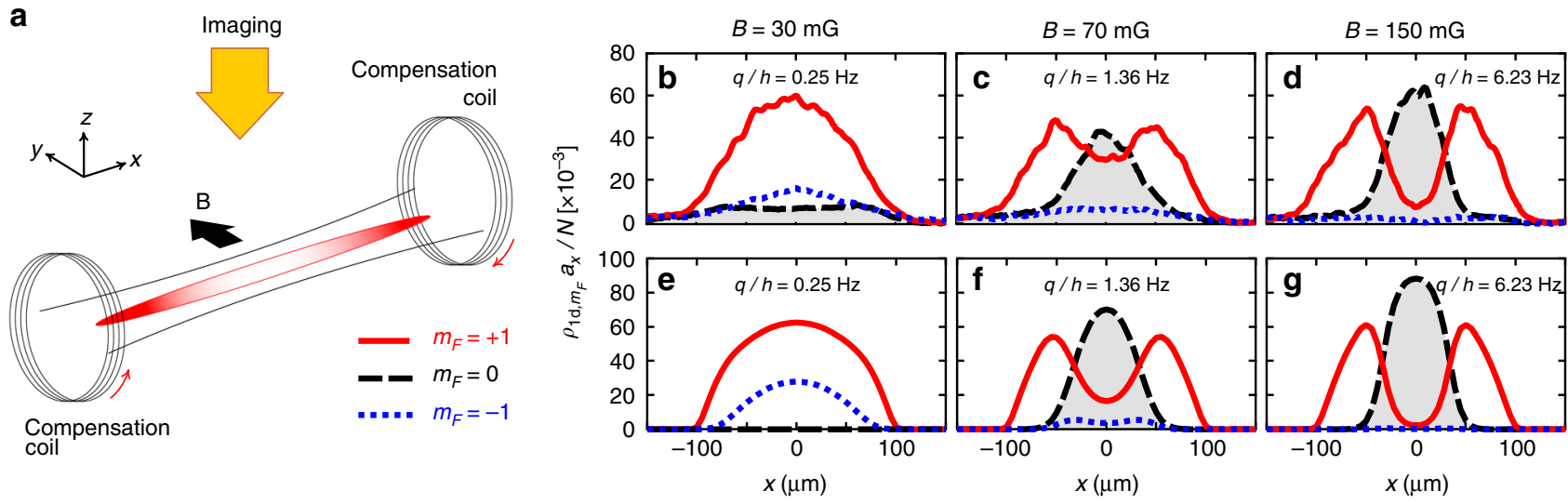

Fig. 1 Spin domain formation in a quasi-1d spinor gas without applied magnetic force. a Experimental setup. A quasi-one-dimensional Bose-Einstein condensate of spin-1 sodium atoms is immersed in a spatially uniform magnetic field $\boldsymbol{B}$. We use the compensation coils to cancel stray magnetic field gradients along the long axis of the cloud, thereby suppressing external magnetic forces. $\mathbf{b}-\mathbf{g}$ Linear density profiles at different magnetic fields B. $\mathbf{b}$-d show the experimental profiles, obtained by averaging over about 100, 40, and 30 individual profiles, respectively. For low-applied magnetic fields, we find that the Zeeman components $m_{F}= \pm 1$ coexist, with $m_{F}=0$ atoms forming a broad, presumably thermal background. When the magnetic field increases, we observe the formation and growth of a $m_{F}=0$ domain at the center of the trap. $\mathbf{e}-\mathbf{g}$ show theoretical profiles at $T=0$, calculated by solving numerically the one-dimensional spin-1 Gross Pitaevskii equation (see Methods), in good agreement with the observed profiles. The average profiles are symmetric under reflection, as expected in the absence of magnetic gradients along $x$. The trap frequencies are $\left(\omega_{x \prime} \omega_{y}, \omega_{z}\right)=2 \pi \times(3.1,270,270) \mathrm{Hz}$, the magnetization is $M_{\|} \approx 0.5 \mathrm{~N}$ and the atom number is $N \approx 10^{4}$

determined by the competition between the interatomic interactions and the Zeeman energy in an applied magnetic field. The total mean-field energy at $T=0$ takes the form ${ }^{3}$

$$
E=\int d \mathbf{r}\left(\boldsymbol{\Psi}^{*} \hat{h} \boldsymbol{\Psi}+\frac{\bar{g}}{2} \rho^{2}+\frac{g_{s}}{2} \mathbf{m}^{2}\right) .
$$

Here, $\hat{h}=-\frac{\hbar^{2}}{2 m_{\mathrm{Na}}} \Delta \cdot++E_{\mathrm{Zeeman}}$ is the single-particle Hamiltonian, $m_{\mathrm{Na}}$ is the atomic mass, $E_{\mathrm{Zeeman}}$ is the Zeeman energy discussed below, and $V=\frac{1}{2} m_{\mathrm{Na}}\left[\omega_{x}^{2} x^{2}+\omega_{y}^{2}\left(y^{2}+z^{2}\right)\right]$ the trapping potential. The partial densities are given by $\rho_{m_{F}}=\left|\Psi_{m_{F}}\right|^{2}$, and the total density by $\rho=\sum_{m=0, \pm 1} \rho_{m}$. The magnetization density $\mathbf{m}$ is defined by its Cartesian components $m_{\alpha}=\sum_{i, j} \Psi_{i}^{*}\left(\hat{S}_{\alpha}\right)_{i, j} \Psi_{j}$, with $\hat{S}_{\alpha}(\alpha=x, y, z)$ the standard spin-1 matrices ${ }^{3}$. We denote by $m_{\|}$the component of $\mathbf{m}$ along the axis of the applied magnetic field $\mathbf{B}$. For instance, for a spin-polarized gas of atoms in $m_{F}=1$, the magnetization density is equal to the total density, $m_{\|}=\rho$, while $m_{\|}=0$ for atoms in $m_{F}=0$.

The two coupling constants $\bar{g}$ and $g_{s}$ characterize spinindependent and spin-dependent interactions, respectively. For sodium atoms in the $F=1$ hyperfine manifold the spindependent interactions are antiferromagnetic $\left(g_{s}>0\right)$, a key feature to observe phase separation ${ }^{30}$. Furthermore, the spindependent term $\propto g_{s}$, although much weaker than the dominant spin-independent term $\left(g_{s} / \bar{g} \approx 0.036\right)$, is essential to understand spinor gases: This term lifts spin degeneracies left by $\bar{g}$ and determines the magnetic properties at very low temperatures.

Spinor gases are typically immersed in a uniform magnetic field $\boldsymbol{B}$ that shifts the internal energy levels by the Zeeman effect. The interaction Hamiltonian conserves the total magnetization $M_{\|}=\int d \mathbf{r} m_{\|}$. As a result, the constant of motion $M_{\|}$should be viewed as an experimental control parameter and not as a dynamical variable. The conservation of $M_{\|}$makes the first-order Zeeman shift linear in $B$ irrelevant to the equilibrium properties. The relevant shift comes from the second-order or quadratic Zeeman energy (QZE), $E_{\text {Zeeman }}=-q \int d \mathbf{r} \rho_{0}$ (up to a constant), with $q=\alpha_{q} \mathbf{B}^{2}$ and $\alpha_{q} \approx h \times 277 \mathrm{~Hz} / \mathrm{G}^{2}$ for sodium atoms. Note that the physics explored in this work does not depend of the orientation of $\mathbf{B}$ with respect to the trap axis, only of its modulus B determining the QZE. This conclusion only holds if dipole-dipole interactions are negligible compared to shortranged van der Waals interactions.

Magnetic phase diagram and spontaneous phase separation. We explore in Fig. $2 \mathrm{a}-\mathrm{c}$ the equilibrium spatial structure of a quasi-1d antiferromagnetic spin-1 Bose gas in a spatially uniform applied field $\mathbf{B}$. We set the total magnetization to $M_{\|} \approx 0.5 \mathrm{~N}$ and vary the QZE $q$. We find that the spatial structure of the spinor gas undergoes a marked change as $q$ increases. For low $q$, we observe a mixed phase where $m_{F}= \pm 1$ domains coexist in the same region of space in the center of the trap, surrounded by magnetized $m_{F}=+1$ regions near the edges of the cloud. Above a critical QZE $q_{1, \exp } \approx h \times 0.72(14) \mathrm{Hz}$ (corresponding to a magnetic field $B_{1, \exp } \approx 51(5) \mathrm{mG}$ ), the $m_{F}=0$ component appears and develops into a domain expelling $m_{F}= \pm 1$ from the central region. The quoted experimental value of $q_{1 \text {,exp }}$ is found by fitting an empirical function-constant below $q_{1 \text {,exp }}$ and growing as $1-$ $e^{-\left|q-q_{1, \text { exp }}\right| / \Delta q}$ above-to the $m_{F}=0$ density in the trap center (Fig. 2b-see also the Supplementary Fig. 5 that shows the behavior of the reduced populations). Error bars denote the $90 \%$ uncertainty level of the fit obtained by standard error analysis assuming Gaussian noise. Furthermore, for $q \gtrsim h \times 2 \mathrm{~Hz}(B \gtrsim 85$ $\mathrm{mG})$, the mixed $m_{F}= \pm 1$ region essentially disappears and the spin-1 gas reduces to a binary mixture of $m_{F}=0,+1$. Our data are summarized in Fig. 2a, where we plot the linear partial densities $\rho_{1 \mathrm{~d}, m_{F}}$ versus $q$. We observe similar behavior for other values of the magnetization $M_{\|}$.

Besides the stripes due to phase fluctuations discussed earlier, we also observe substantial position fluctuations of the spin domains. For instance, in the examples shown in Fig. 1d, we find that the center-of-mass of the $m_{F}=0$ component fluctuates by $\sim 16 \mu \mathrm{m}$ for $B=45 \mathrm{mG}$ and by $\sim 6 \mu \mathrm{m}$ for $B=150 \mathrm{mG}$. We believe that this behavior is due to thermal fluctuations of the domain, and not to a technical artifact such as a magnetic gradient fluctuating around the compensated value. The fluctuations of the 

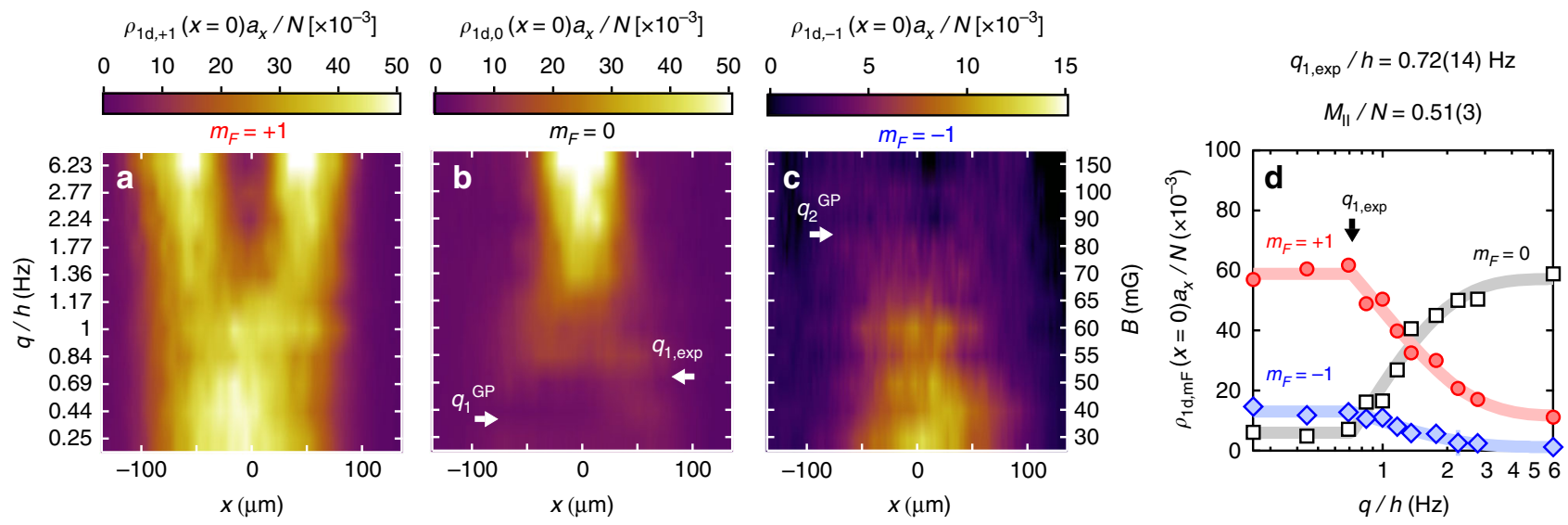

Fig. 2 Formation of equilibrium spin domains without magnetic force. a-c Linear densities of each Zeeman component $m_{F}$ as a function of position for increasing quadratic Zeeman energies $q$. Each line in the false color plots corresponds to an individual profile as shown in Fig. 1b. The arrows mark the locations of the observed $q_{1, \exp }$ and predicted $q_{1}^{\mathrm{GP}}, q_{2}^{\mathrm{GP}}$ critical quadratic Zeeman energies. $\mathbf{d}$ Magnetic transition for $M_{\|} / N=0.51$ (3). Evolution with $q$ of the normalized densities at the center of the trap. The gray lines show a piece-wise function constant below $q_{1, \exp }$ and growing as $1-e^{-\left|q-q_{1, \text { exp }}\right| / \Delta q}$ above. We obtain the quoted experimental values of $q_{1, \text { exp }}$ fitting this function to the experimental data for $m_{F}=0$. The quoted uncertainty correspond to a $90 \%$ confidence interval assuming Gaussian noise and independent errors. The trap frequencies, magnetization, and atom number are as in Fig. 1

position of the spin domains and their possible use for lowtemperature thermometry will be explored in more detail in a future publication.

Phase coexistence in homogeneous systems. The observed characteristics of the phase diagram can be qualitatively understood by considering first a uniform system in the thermodynamic limit enclosed in a box of volume $\mathcal{V}$. Three homogeneous phases can be realized depending on the magne-

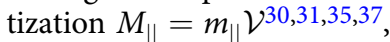

Phase I or Unmagnetized phase: All atoms occupy the $m_{F}=0$ Zeeman state, with $m_{\|}=0$ and $\rho_{0}=\rho$,

Phase II or Partially magnetized phase: The components $m_{F}=$ \pm 1 coexist, with magnetization density $0<m_{\|}<\rho$ and $\rho_{0}=0$,

Phase III or Fully magnetized phase: All atoms occupy the $m_{F}=+1$ Zeeman state, with $m_{\|}=\rho$ and $\rho_{0}=0$. Note that phase II evolves continuously into phase III when the magnetization increases.

The properties of the various phases are summarized in Table 1. A completely homogeneous phase where the three Zeeman components coexist is always unstable toward phase separation $^{35}$. For a partially magnetized system with $0<M_{\|}<N$, phase II is the only possible homogeneous phase compatible with the conservation of the total magnetization $M_{\|}$. However, it competes with inhomogeneous (phase-separated) configurations, either I-II or I-III, depending on the value of $q^{35}$.

A common choice in the literature $e^{30,35}$ is to describe the evolution of the system for fixed $\rho, m_{\|}$, and with varying QZE $q$. For low QZE, phase II minimizes the interaction energy and is the stable equilibrium phase. As the QZE increases, a mixed configuration where part of the system is in phase II and part in phase I becomes energetically competitive. The preferred equilibrium configuration can be determined by comparing the energies of the competing possibilities (neglecting the energy cost of the I-II interface),

$$
\delta E=E_{\mathrm{I}-\mathrm{II}}-E_{\mathrm{II}}=\mathcal{V} f_{0} \times\left[\frac{g_{s} m_{\|}^{2}}{2\left(1-f_{0}\right)}-q \rho\right],
$$

with $f_{0}$ the fraction of the available volume occupied by phase I in the mixed configuration. When $q \geq q_{1}=g_{s} m_{\|}^{2} /(2 \rho)$, $\delta E$ becomes

\begin{tabular}{|c|c|c|c|}
\hline Phase & I $\left(m_{F}=0\right)$ & II $\left(m_{F}= \pm 1\right)$ & III $\left(m_{F}=+1\right)$ \\
\hline $\begin{array}{l}\text { Spin density } \\
\text { Energy } \\
\text { density }\end{array}$ & $\begin{array}{l}m_{\|}=0 \\
\frac{\bar{g}}{2} \rho^{2}-q \rho\end{array}$ & $\begin{array}{l}m_{\|}<\rho \\
\frac{\bar{g}}{2} \rho^{2}+\frac{g_{s}}{2} m_{\|}^{2}\end{array}$ & 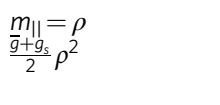 \\
\hline $\begin{array}{l}\text { Equation of } \\
\text { state }\end{array}$ & $\mu+q=\bar{g} \rho$ & $\begin{array}{l}\mu=\bar{g} \rho, \eta= \\
g_{\mathrm{s}} m_{\|}\end{array}$ & $\mu+\eta=\left(\bar{g}+g_{s}\right) \rho$ \\
\hline
\end{tabular}

The table summarizes the thermodynamic properties of the three stable homogeneous phases for uniform system in the thermodynamic limit

negative for $f_{0}=0$ and the homogeneous phase II becomes thermodynamically unstable. Above $q_{1}$, a phase I domain forms. The equilibrium fraction of $m_{F}=0$ atoms grows as $f_{0}\left(q \geq q_{1}\right)=1$ $-\left(q_{1} / q\right)^{1 / 2}$. The conservation of the total magnetization $M_{\|}$then requires that the magnetization density in subregion II decreases as $m_{\|}=M_{\|} /\left[\mathcal{V}\left(1-f_{0}\right)\right]$. When $m_{\|}=\rho \quad\left(f_{0}=1-M_{\|} / N\right)$, one obtains a phase-separated I-III mixture which remains the same when $\rho$ increases further. The sequence of transitions is illustrated in Fig. 3a.

Anticipating the discussion of the trapped case within the framework of the local density approximation (LDA), we now adopt a slightly different point of view and consider the properties of the system for fixed $q$ and varying $\rho, m_{\|}$(Fig. 3b). It is convenient to chose a thermodynamic ensemble characterized by two thermodynamic potentials enforcing the conservation of $N$ and of $M_{\|}$, respectively: the chemical potential $\mu$ and another potential $\eta$ (that could be called the thermomagnetic potential). The equation of state of the various phases are given in terms of $\mu$ and $\eta$ in Fig. 3a. Phase II (respectively phase I) is the stable equilibrium phase for densities below (resp. above) a critical value defined by

$$
(\rho q)_{1}=\frac{\eta^{2}}{2 g_{s}}=\frac{g_{s} m_{\|}^{2}}{2}
$$

where $\eta=g_{s} m_{\|}$in phase II. A second, continuous II-III transition 
a

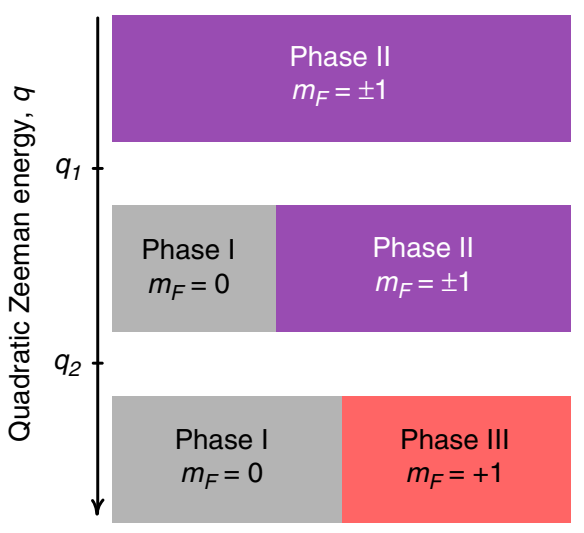

b

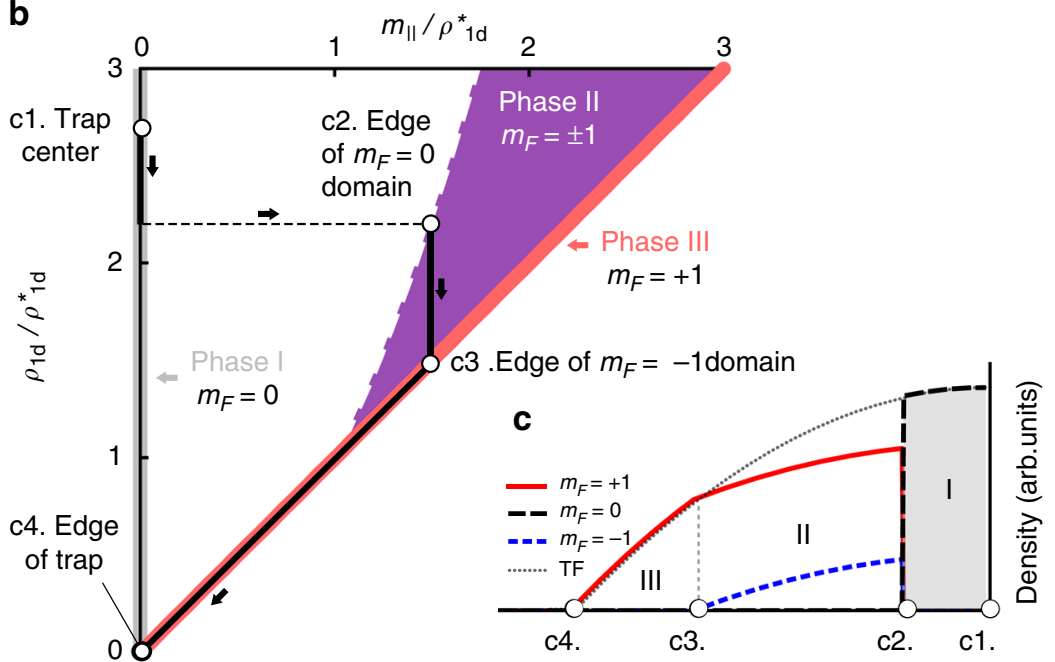

Fig. 3 Phase diagram of homogeneous spin-1 systems. a Phase diagram for fixed particle and magnetization densities $\rho$ and $m_{\|}$as a function of the quadratic Zeeman energy q. b Phase diagram at constant quadratic Zeeman energy $q$, as a function of $\rho, m_{\|}$. The density scale is $\rho^{\star}=2 q / g_{\mathrm{s}}$. The black line and the arrows show a typical trajectory from the trap center to the cloud edge for a trapped system treated in the local density approximation. For the particular example shown in the figure, phase I is realized near the center of the cloud, and the state of the system jumps from phase I to phase II along the dashed line when the density reaches a critical value. c Density profiles of the Zeeman components in a trap in the local density approximation, corresponding to the trajectory shown in $\mathbf{b}$. The dotted line shows the total density

occurs at $m_{\|}=\rho^{*}$, with the characteristic density

$$
\rho^{*}=\frac{2 q}{g_{\mathrm{s}}}
$$

with the fully magnetized phase III realized for densities lower than $\rho^{*}$.

Spatial structure of a trapped system. The preceding discussion is directly relevant to determine the spatial structure of a quasi-1d gas in a harmonic trap where $\rho, m_{\|}$, and $\rho_{0}$ vary with position. We consider in this Section the purely $1 \mathrm{~d}$ limit $\mu \ll \hbar \omega_{y}$ where the transverse motion is frozen in the transverse ground state of the trap. We first perform our analysis within the LDA, and discuss effects beyond the LDA at the end of this section. The equalities established in the previous section remain valid substituting linear densities $\rho \rightarrow \rho_{1 \mathrm{~d}}$ and $\left(\bar{g}, g_{\mathrm{s}}\right) \rightarrow\left(\bar{g}^{1 \mathrm{~d}}, g_{\mathrm{s}}{ }^{\mathrm{ld}}\right)$, with effective $1 \mathrm{~d}$ coupling constants $\left(\bar{g}^{1 \mathrm{~d}}, g_{\mathrm{s}}^{1 \mathrm{~d}}\right)=\left(\bar{g}, g_{\mathrm{s}}\right) \times 1 /\left(2 \pi a_{y}^{2}\right)$. Here, $a_{y}=$ $\sqrt{\hbar /\left(m_{\mathrm{Na}} \omega_{y}\right)}$ is the transverse harmonic oscillator size. We keep the same notation $m_{\|}$for the integrated magnetization density with a slight abuse of notation. The pure $1 \mathrm{~d}$ limit is not strictly realized in our experiment, as noted earlier. However, we have evaluated corrections to this limit and found that they only change marginally the conclusions (see Supplementary Fig. 4 and Methods). As a result we stick to the $1 \mathrm{~d}$ description in the core of the article to keep the discussion as simple as possible.

In general, one can find three regions corresponding to the three homogeneous phases. Moving from the edges to the center, phase III first appears for low densities in a region where $0 \leq \rho_{1 \mathrm{~d}}(x) \leq \rho_{1 \mathrm{~d}}^{*}$, followed by phase II where $\rho_{1 \mathrm{~d}}^{*} \leq \rho_{1 \mathrm{~d}}(x) \leq \rho_{c 2}$, and eventually by phase I near the trap center where $\rho_{1 \mathrm{~d}}(x) \geq \frac{m_{\|}^{2}}{\rho_{1 \mathrm{~d}}^{*}}$. The magnetization density $m_{\|}$is zero in region $\mathrm{I}$, equal to $\eta / g_{\mathrm{s}}^{1 \mathrm{~d}}$ and spatially uniform in region II, and equal to the total density (and therefore nonuniform) in region III. A corresponding trajectory in the $\left(\rho, m_{\|}\right)$plane is shown in Fig. 3c.
For given $N, M_{\|}$the condition for the appearance of phase I in the center of the trap given in Eq. (3) can only be fulfilled for sufficiently high QZE $q$. Similarly to the homogeneous case, this leads to a first critical value $q_{1}$ that corresponds to our measured $q_{1, \exp }$. The magnetization density $m_{\|}$in region II is uniform, but not directly proportional to $M_{\|}$as it was in the homogeneous case. For a purely $1 \mathrm{~d}$ system, we find following ref. ${ }^{46}$ that $m_{\|} \approx$ $\rho_{1 \mathrm{~d}}(0)\left[1-\left(1-\left(M_{\|} / N\right)\right)^{2 / 3}\right]^{2}$ for $q \leq q_{1}$. Using Eq. (3), this gives the LDA prediction for the first critical QZE ${ }^{46}$,

$$
q_{1, \mathrm{LDA}}=\frac{g_{\mathrm{s}} \mu}{2 \bar{g}}\left[1-\left(1-\frac{M_{\|}}{N}\right)^{2 / 3}\right]^{2}
$$

Using our experimental parameters $(\mu / h \approx 120 \mathrm{~Hz})$, we obtain $q_{1}$, LDA $\approx h \times 0.3 \mathrm{~Hz}$, substantially below the observed $q_{1 \text {,exp }} \approx h \times$ $0.72 \mathrm{~Hz}$. The same conclusion holds when taking the deviations from the purely $1 \mathrm{~d}$ case into account (see Supplementary Methods).

The quantitative difference between the observations and the LDA prediction can be expected, as the latter completely neglects the energy cost of the interface between two immiscible phases. This cost comes from the balance between the kinetic energy, increasing when the width of the interface decreases, and the interaction energy, increasing when the interface spreads out due to the increased overlap between the two components. The interface energy is proportional to its width (typically several times the spin healing length) $\left(\zeta_{s}=\sqrt{\hbar^{2} \bar{g} /\left(2 m_{\mathrm{Na}} g_{\mathrm{s}} \mu\right)} \sim 7 \mu \mathrm{m}\right)$, and therefore not extensive and negligible for infinitely large systems. However it can be significant in a gas of finite extent as in our experiment where a typical cloud half-length is $L=\sqrt{2 \mu /\left(m \omega_{x}^{2}\right)} \sim 100 \mu \mathrm{m}$.

These effects beyond the LDA can be explored at zero temperature using the mean-field theory of spin-1 gases, which takes the form of three coupled Gross-Pitaevskii equations. We have solved these equations numerically to find the lowest energy solution (see Methods). Examples of the density profiles that we obtain numerically are shown in Fig. 1e-g. Using the same fitting 
procedure as for the experimental data in Fig. 2d, we find that the first critical QZE predicted by the GP approach is $q_{1}^{\mathrm{GP}} \approx h \times 0.36 \mathrm{~Hz}$. Therefore the discrepancy between the measured and predicted first critical QZE is not resolved by upgrading the theory from LDA to GP. A second critical QZE $q_{2}^{\mathrm{GP}} \approx h \times 2 \mathrm{~Hz}$ where $m_{F}=-1$ disappears can also be identified in the GP calculation. This is consistent with the experimental results, although we find experimentally that the population of the $m_{F}=-1$ component decreases smoothly with $q$ and does not completely vanish at high $q$. This prevents us to clearly identify a critical value $q_{2, \exp }$ analogous to $q_{2}^{\mathrm{GP}}$.

To summarize, we used the LDA to describe the spatial structure of a trapped gas in terms of the phase diagram of the uniform system at $T=0$. We were able to account qualitatively for the observations, but found quantitative differences. In particular, the measured critical field where spin domains appear is higher than predicted. In the next section, we show that the discrepancy between the measured $q_{1}$ and the $T=0$ prediction, as well as the difficulty in identifying $q_{2}$ in experiments, can be understood qualitatively by considering the role of a finite temperature of the sample.

Role of the thermal components. To compare the experimental results with the prediction of the spin-1 GP theory in more detail and discuss the role of a thermal component, we define an effective size for each Zeeman component as the root-meansquare (rms) radius restricted to the condensate region $[-L, L]$,

$$
R_{m_{F}}=\frac{1}{\mathcal{N}_{m_{F}}} \int_{-L}^{L} x^{2} \rho_{1 \mathrm{~d}, m_{F}}(x) d x,
$$

where the half-length $L$ of the condensate is found by a parabolic fit to $\rho_{1 \mathrm{~d}}(x)$, and with a normalization factor $\mathcal{N}_{m_{F}}=\int_{-L}^{L} \rho_{m_{F}, 1 \mathrm{~d}}(x) d x$. We show in Fig. $4 \mathrm{a}-\mathrm{c}$ the size $R_{m_{F}}$ computed from the measured profiles and from the calculated ones. The size of $m_{F}=+1$ increases only slightly with $q$, and stays close to the $T=0 \mathrm{GP}$ prediction for all values of $q$. In contrast, both $R_{m_{F}=0}$ and $R_{m_{F}=-1}$ differ substantially from the $T=0$ predictions. Focusing on $m_{F}=0$, the rms radius starts from a large value at low $q$, then decreases above $q_{1, \exp }$ before settling to an asymptotic value above $q \gtrsim h \times 2 \mathrm{~Hz}$. The agreeement between experiments and $T=0$ theory improves with increasing $q$.

The differences between experiment and theory can be explained qualitatively by thermal excitations. Low-energy excitations of homogeneous spin-1 BECs have been studied using the Bogoliubov approach ${ }^{2,3,53}$. In general, one expects for $q \neq 0$ that the Bogoliubov spectrum consists of three modes. For low values of $q \ll q_{1}$, where the (quasi-)condensate occupies the $m_{F}= \pm 1$ states, one spin mode essentially reduces to excitations of atoms in the $m_{F}=0$ state with a gap $E_{g} \geq q^{2}$. In a Hartree-Fock picture appropriate for $k_{B} T \gg g_{s} \rho$, the effective potential seen by the uncondensed $m_{F}=0$ atoms is almost flat (up to small terms $\left.\propto g_{s}\right)$ : The mean-field from the condensate in $m_{F}= \pm 1$ cancels almost exactly the trapping potential ${ }^{54,55}$. Uncondensed excitations in $m_{F}= \pm 1$ experience a different mean-field potential that expels them from the trap center. As a result one expects that below $q_{1}$ the thermal component occupies mostly the $m_{F}=0$ Zeeman state. In Fig. 4d, we show a magnified view of the linear density profiles for $q<q_{1, \exp }$. A subtantial population is present in $m_{F}=0$ (in contrast to the $T=0$ prediction, see Fig. $4 \mathrm{e}$ ) and shows a flat-top profile within the volume where the $m_{F}= \pm 1$ condensate is present, in agreement with the Hartree-Fock description. For a flat density confined within the condensate region $[-L, L]$, the rms radius is $\approx \sqrt{1 / 3} L \approx 62 \mu \mathrm{m}$, in good agreement with the measured $R_{m_{F}=0} \approx 58 \mu \mathrm{m}$ for low $q$.
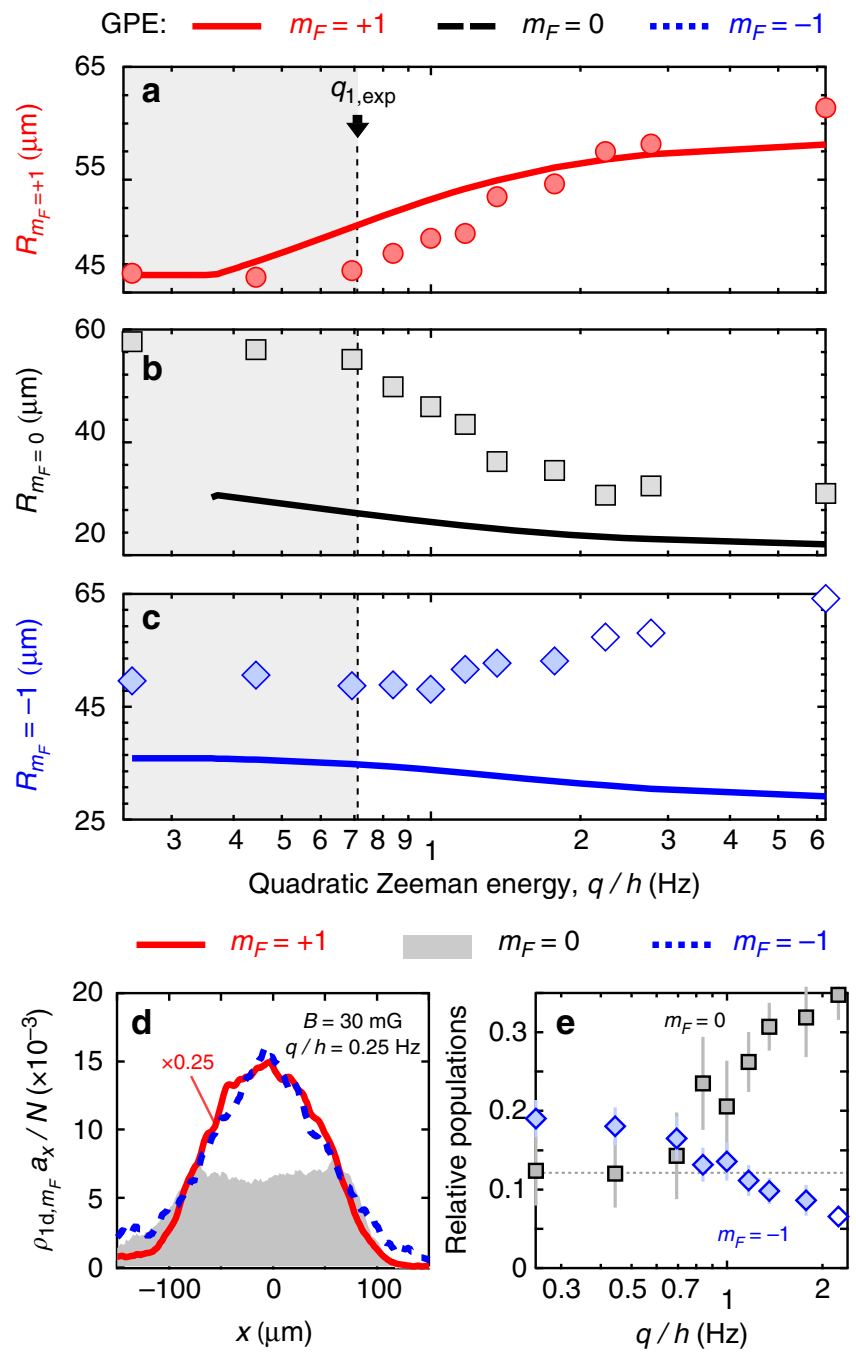

Fig. 4 Domain size and role of the thermal component. a-c Measured (symbols) and calculated (lines) root-mean-square sizes $R_{m_{f}}$ of each Zeeman component versus $q$. Open symbols indicate situations where the atomic density in $m_{F}=-1$ becomes comparable to the imaging background noise (total population below $\sim 700$ atoms). We ascribe the differences between the measured and calculated $R_{m_{F}=0,-1}$ to the presence of a substantial thermal component. d Density profiles of the $m_{F}=0$ (solid, shadowed region), $m_{F}=+1$ (continuous curve), and $m_{F}=-1$ components (dashed curve) below $q_{1, \text { exp }}$. The $m_{F}=0$ component exhibits a flat-top profile within the condensate region. The profile is the same as in Fig. 1b, $q / h=0.25 \mathrm{~Hz}$, with the $m_{F}=+1$ component scaled down by a factor 0.25 for clarity. e Total populations of the $m_{F}=0$ and $m_{F}=-1$ components $(q / h$ $=0.25 \mathrm{~Hz}$ ). Error bars indicate the empirical standard deviations of the data calculated over several tens of profiles for each $q$. The trap frequencies, magnetization, and atom number are shown in Fig. 1b-d

This discussion, although qualitative, explains the increase of the observed critical field from the $T=0$ value. For $q \gtrsim q_{1}$, the small domain expected at $T=0$ does not actually form but rather dissolve inside the existing $m_{F}=0$ thermal component. The suppression of phase separation at finite temperatures has been noted in a theoretical study of a two-component gas ${ }^{56}$, and is also consistent with our previous experimental work on threedimensional spin-1 gases ${ }^{57}$. We are not aware of theoretical studies of antiferromagnetic spin-1 gases in 1d that can explain our observations quantitatively. Our experiments could be modeled using classical field methods (see ref. ${ }^{58}$ for a review) 
and perhaps used to benchmark such methods. To ease such comparison, we have measured the temperature of the thermal component by fitting the equation of state obtained from the wings of the linear profiles ${ }^{59}$ to a Hartree-Fock model of our quasi-1 gas $^{60}$. Here, the wings correspond to the nondegenerate region of the cloud where the one-dimensional phase space density $\rho_{1 \mathrm{~d}} \lambda_{T} \leq 1$, with $\lambda_{T}=\sqrt{2 \pi \hbar^{2} /\left(m_{\mathrm{Na}} k_{B} T\right)}$ the thermal De Broglie wavelength and $k_{B}$ the Boltzmann constant. We find $T \approx 30-40 \mathrm{nK}$ without any obvious dependence on $q$. Note that the measured temperature is substantially higher than the spindependent energies $\eta, q$ explored in this work. Hence, even if quantum and thermal fluctuations probably lead to qualitatively similar effects on the domain formation, we expect the latter to be dominant in our experimental conditions.

Long-time relaxation of out-of-equilibrium spin textures. Having characterized the equilibrium properties of a spin-1 antiferromagnetic gas, we now turn to nonequilibrium behavior. We investigate how an initial, highly nonequilibribrium configuration relaxes to a final equilibrium configuration. The experiment is performed at a uniform bias field $B=600 \mathrm{mG}(q / h \approx 100$ $\mathrm{Hz}$ ), well above $q_{2}^{\mathrm{GP}}$. We prepare the system at a magnetization $M_{\|} \approx 0.66(2) N$ using the same procedure as before, except for an applied magnetic potential $V_{\mathrm{mag}}=g_{F} m_{F} \mu_{B} b^{\prime} x$ along $x$ controlled by an applied magnetic gradient $b^{\prime}$ ( $\mu_{B}$ is the Bohr magneton and $g_{F}=-1 / 2$ the Landé $g$-factor). Using $b^{\prime}=24 \mathrm{mG} / \mathrm{cm}$, the net effect of the combined action of the magnetic force and of spindependent interactions is to pull the $m_{F}=+1$ Zeeman component to the right side of the cloud while pushing the $m_{F}=0$ component to the left one. Atoms in $m_{F}=-1$ are purely thermal and barely discernible in this regime.

We remove the applied magnetic force at $t=0$, leaving the spin-1 gas in a purely optical potential independent of the Zeeman state but also in a highly nonequilibrium configuration. The first consequence is an excitation of the center-of-mass (c.o. m.) motion of the cloud that persists up to $20 \mathrm{~s}$, the longest time we explored (see Fig. 5a). This motion is common-mode to the $m_{F}=0$ and +1 components and occurs at the expected dipole mode frequency $\omega_{x}$. In contrast, the relative positions of the two Zeeman components do not display any detectable oscillation and evolve on a much longer time scale than the axial period, as pictured in Fig. 5b. To quantify the relaxation we introduce the c.o.m. displacements

$$
\Delta \bar{x}_{m_{F}}=\frac{1}{N_{m_{F}}} \int(x-\bar{x}) \rho_{1 \mathrm{~d}, m_{F}}(x) d x,
$$

of the $m_{F}=+1$ and $m_{F}=0$ components from the center of mass $\bar{x}=(1 / N) \int x \rho_{1 \mathrm{~d}}(x) d x$ of the whole cloud. Here, $N_{m_{F}}=$ $\int \rho_{1 \mathrm{~d}, m_{F}}(x) d x$ is the total population of the $m_{F}$ component. We report in Fig. $5 c$ the relative displacement of $m_{F}=0$, which remains mostly constant for several periods of the c.o.m. oscillations before decaying to zero within a timescale of $\sim 4 \mathrm{~s}$.

The profiles shown in Fig. $5 \mathrm{~b}$ indicate that this relaxation occurs progressively, with the $m_{F}=0$ component penetrating slowly into the $m_{F}=+1$ majority component. This behavior could be surprising for a truly immiscible binary mixture, where the repulsion between the species acts as an effective barrier preventing relaxation. Figure $5 \mathrm{~d}$ shows that the $m_{F}=-1$ component, altough weakly populated, plays an important role in the relaxation process. The relative populations of the Zeeman states evolve on the same time scale as the relaxation, with a decrease in the population of $m_{F}=0$ and a roughly equal increase in the populations of $m_{F}= \pm 1$. This indicates that spin-changing collisions of the form $2 \times\left(m_{F}=0\right) \rightarrow\left(m_{F}=\right.$ $+1)+\left(m_{F}=-1\right)$ are involved in the mechanism enabling $m_{F}=0$ atoms to cross the effective energy barrier due to spin-dependent interactions.

The process is most likely dominated by excitations residing initially in the inferface between the $m_{F}=0$ and $m_{F}=+1$ regions, and seeding the long-time dynamics ${ }^{33}$. The effective mean-field potential experienced by atoms in $m_{F}=-1$ inside the volume of the $m_{F}=+1$ domain is not exactly flat but slightly attractive, $V_{\mathrm{eff}}(x)=\frac{1}{2} M_{\mathrm{Na}} x^{2}+\bar{g} \rho(x)-g_{\mathrm{s}} \rho_{+1}(x) \approx \mu-g_{\mathrm{s}} \rho_{+1}(x)$. Atoms in $m_{F}=-1$ created in the interface by the process $2 \times\left(m_{F}=0\right) \rightarrow$ $\left(m_{F}=+1\right)+\left(m_{F}=-1\right)$ can thus freely migrate toward the trap center, where they can recombine with $m_{F}=+1$ atoms to generate $m_{F}=0$ atoms by the inverse process. This mechanism effectively enables $m_{F}=0$ atoms to crawl through the otherwise impenetrable $m_{F}=+1$ component. This mechanism could be coherent (preserving the initial relative phase), but need not be. Given that $T \gg g_{s} \rho$, we believe it is likely that the relaxation process is seeded by the thermal component initially present near the interface.

A recent experiment by Eto et al. ${ }^{61}$ appears similar at first sight, but reports drastically different results: bouncing of the spin components against each other in the strongly immiscible regime, followed by relaxation in a few hundreds of ms to a highly excited state with large kinetic energy. We believe the mechanism at play in our experiments is different from the ones studied in ref. ${ }^{61}$, where the experimental parameters differ by orders of magnitude. Eto et al. apply a bias field of $11 \mathrm{G}$ which supresses completely spin-changing collisions (we use $600 \mathrm{mG}$ ), and a gradient of $900 \mathrm{mG} / \mathrm{cm}$ (we use $10 \mathrm{mG} / \mathrm{cm}$ ). It is then not really surprising that we do not observe the bouncing motion, nor the formation of stripes reported by Eto et al. in the strongly immiscible regime.

Figure 5e displays histograms of the c.o.m. of the $m_{F}=0,+1$ components as a function of the relaxation time. We observe a gradual change over time from a distribution peaked near the cloud edges to a distribution peaked near the cloud center. The distribution of $\bar{x}_{0}$ appears smooth and single-peaked at all times. These observations rule out a scenario where relaxation is explained by a macroscopic quantum tunneling of the $m_{F}=0$ component. In that case, we expect at intermediate times that the $m_{F}=0$ component is in a superposition of two domains, one localized on the left side of the $m_{F}=+1$ cloud and one localized near its center. This would lead to a bimodal spatial distribution for which we find no evidence.

\section{Discussion}

Magnetically ordered many-body systems characterized by nonuniform order parameters are plentiful. The simplest example corresponds to antiferromagnetism of localized spins (here the adjective localized means that the mobility of the spincarrying particles is irrelevant, and that the physics reduces to a pure spin problem). A second, richer case occurs in itinerant magnetism when the spin-carrying particles are mobile. Stripe phases of electrons in strongly correlated materials are prominent examples $^{62}$. These materials are hole-doped antiferromagnets, which organize for certain doping levels in antiferromagnetic domains separated by nanometer-size conducting domain walls. This structure is believed to arise from the competition between the kinetic energy of the holes and the exchange interactions between the spins. This example illustrates that one can expect (and often finds) nonuniform phenomena in the magnetism of itinerant quantum particles. In this article, we have investigated the formation and relaxation of inhomogeneous spin domains in a quasi-1d spin $F=1$ Bose gas with 
a
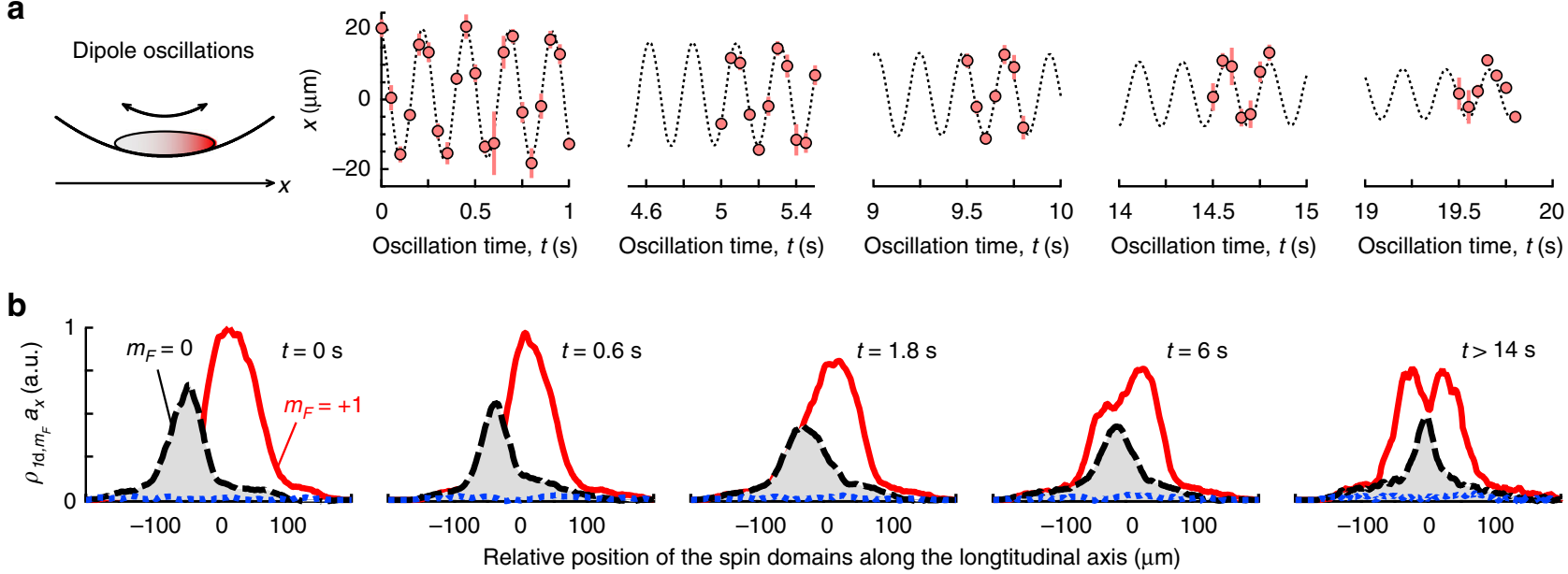

C
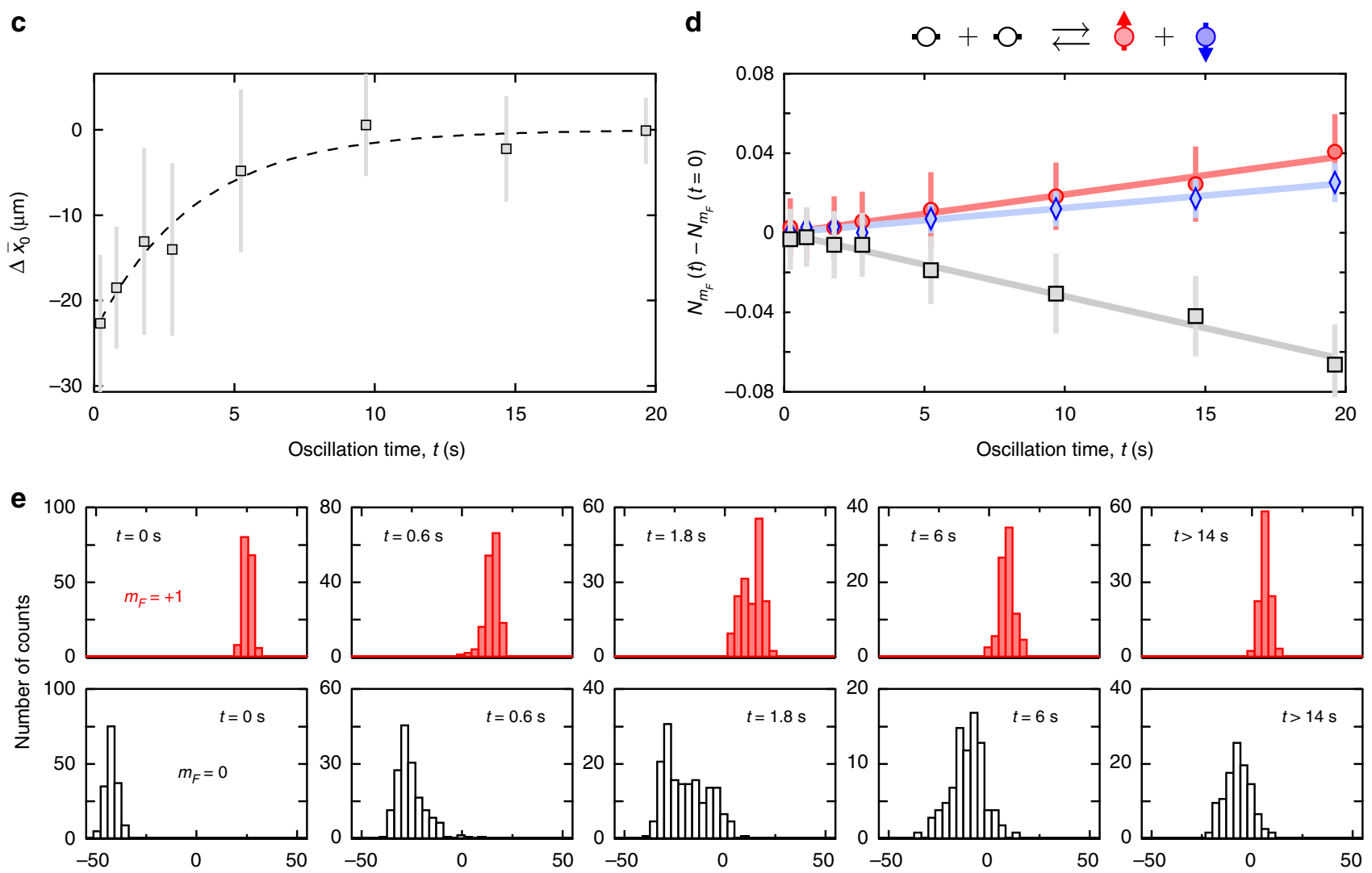

Relative position of the spin domains along the longitudinal axis $(\mu \mathrm{m})$

Fig. 5 Relaxation of spin domains to their equilibrium configuration. a Long-lived center-of-mass oscillations of a partially magnetized gas with $M_{\|} / N \approx$ $0.66(2)$ along the longitudinal trap axis. $\mathbf{b}$ Relaxation to the final equilibrium configuration. An initially asymmetric equilibrium state prepared with $b^{\prime} \neq 0$ relaxes to a symmetric equilibrium state expected for $b^{\prime}=0$ when the gradient $b^{\prime}$ is suddently turned off. The relaxation is much slower than the axial trap period and spans several seconds. $\mathbf{c}$ Long time-scale relaxation dynamics. Evolution of the center-of-mass $\Delta \bar{x}_{0}$ of the $m_{F}=0$ component relative to the center-of-mass of the whole cloud. An exponential fit to the data (dashed line) returns a 1/e relaxation time of $\tau \approx 3.7(1.7)$ s. d. Role of spin-changing collisions. Relative change of the spin populations, $N_{m_{F}}(t)-N_{m_{F}}(0)$ (squares: $m_{F}=0$, circles: $m_{F}=+1$, diamonds: $m_{F}=-1$ ), indicating spin-changing dynamics is involved in the relaxation process. The straight lines are a guide to the eye. e Probing relaxation dynamics from the motion of the $m_{F}=+1$ (top) and $m_{F}=0$ (bottom) components relative to the center-of-mass of the whole cloud. Histograms of the relative displacement $\Delta \bar{x}_{0}$. Each histogram contains at least 170 measurements. For these measurements, the trap frequencies are $\left(\omega_{x}, \omega_{y}, \omega_{z}\right)=2 \pi \times(4.3,385,385) \mathrm{Hz}$ and the atom number is $N \approx 9400$. Error bars in $\mathbf{c}$, $\mathbf{d}$ indicate the empirical standard deviations of the data

antiferromagnetic interactions. The low-field configuration is a mixed phase of the $m_{F}= \pm 1$ components stabilized by antiferromagnetic spin-exchange interactions. An applied uniform bias magnetic field favors the appearance of $m_{F}=0$ atoms through the QZE shift. The two influences compete agains each other, and this competition leads to a critical value of the QZE above which $m_{F}=0$ atoms appear and spontaneously organize in a spin domain near the trap center. We experimentally measured the critical value $q_{1 \text {,exp }}$ where a $m_{F}=0$ domain appears and characterized the phase diagram in detail. 
We found that the $T=0$ mean-field theory of spin-1 Bose gases describes qualitatively well our observations. However, there exist discrepancies between the predicted and measured values of the critical fields. The finite temperature of our samples, although very low, could explain these discrepancies. Indeed, energy scales in spinor gases are naturally low in comparison to the natural scale set by the chemical potential of the BEC. Therefore, we expect that thermal fluctuations are able to suppress the formation of spin domains near the transition where different spin configurations are close in energy. The quasi-1D nature of our experimental system may further enhance thermal effects.

Finally, we studied the nonequilibrium dynamics and relaxation of spin domains in the phase-separated, high- $q$ regime. In contrast to the miscible regime ${ }^{14,63}$, we observe no spin-dipole oscillations in the phase-separated regime. Instead we find that spin dynamics is frozen on short time scales on the order of the trap period, and undergoes slow relaxation toward an equilibrium configuration on long times scales of several tens of axial trap periods (about $10 \mathrm{~s}$ ). We found evidence that relaxation takes place through spin-changing collisions, enabling atoms from immiscible Zeeman components to pass through the effective barrier created by mean-field interactions with the other component. Our results could be explained by a thermally-assisted process where a scarcely populated, but not empty thermal component in $m_{F}=-1$ seeds the relaxation dynamics. We found no clear evidence of macroscopic quantum tunneling.

\section{Methods}

Optical dipole trap. Our experiments start with a spinor gas of ultracold ${ }^{23} \mathrm{Na}$ atoms with a fixed total magnetization $M_{\|}$and immersed in a uniform magnetic field $\mathbf{B}$. The spinor gas is held in a crossed dipole trap (CDT) created at the intersection of two Gaussian beams propagating along the $x$ - and $z$-axes. We prepare a normal gas with a well-defined magnetization far above the critical temperature using spin-distillation in an applied magnetic field gradient at the beginning of the evaporation ${ }^{64,65}$. We then cool the sample to degeneracy using standard evaporative cooling, and obtain a three-dimensional condensate in the CDT with $>90 \%$ condensate fraction. We transfer the condensate to the $1 \mathrm{~d}$ trap by adiabatically turning off one of the dipole beams in $5 \mathrm{~s}$ (see Supplementary Fig. 1 and Methods for more details).

Stern-Gerlach imaging. We measure the density profiles of each Zeeman component by removing suddenly the trapping potential and letting the cloud expand for a time-of-flight (t.o.f.) of $t=8 \mathrm{~ms}$ in a magnetic field gradient (applied only during the t.o.f.). We extract linear density profiles from the two-dimensional absorption images (Supplementary Methods and Supplementary Fig. 2). Owing to the large trap anisotropy, the expansion is essentially in the radial direction (At $T$ $=0$, the condensate expands along its weak axis by a factor $\approx 10^{-466}$ ). The finite width $\zeta$ of the spin domains entails a finite quantum confinement energy. When the gas is released from the trap, the domains are therefore expected to expand at a speed $\sim \hbar /\left(m_{\mathrm{Na}} \zeta\right)^{33}$. However, in our experiments we have $\hbar t /\left(m_{\mathrm{Na}} \zeta\right) \approx 1-2 \mu \mathrm{m} \ll \zeta$, so that we can safely neglect this expansion.

Magnetic field generation. We generate uniform magnetic fields using three pairs of bias coils aligned along the $x \pm y$ and $z$-directions. We calibrate the magnetic fields using radio-frequency spectroscopy, with a typical resolution of $\sim 1 \mathrm{mG}$. We observe magnetic field fluctuations with $\delta B \sim 3 \mathrm{mG}$ root-mean-square (r.m.s.) amplitude and with a typical time scale of several tens of seconds. These fluctuations, coming from a nearby subway line, are along the vertical $z$-axis, orthogonal to the applied bias field $\mathbf{B}$ that lies in the $x-y$ plane. The impact of magnetic field fluctuations is minimized by working with applied fields $B \geq 30 \mathrm{mG}$. The resulting r.m.s. uncertainty on $q$ is then below $\delta q \sim(\delta B / B)^{2} \sim 1 \%$.

Magnetic force cancellation. Our experiments are performed after carefully canceling stray magnetic field gradients (thereby canceling magnetic forces) along the weak axis of the trap. Stray gradients have at least two origins: (i) the residual ambient gradients (arising from inhomogeneously magnetized elements around the experiment, power supplies, etc...) and (ii) the imperfections of the bias coils that produce slightly inhomogeneous fields.

We cancel the residual magnetic force along $x$ by two methods, either by applying a compensation gradient along the weak axis of the trap (more appropriate at low bias fields where effect (i) dominates), or by choosing the direction of the applied field (more appropriate at large bias fields where effect (ii) dominates) [see Supplementary Methods and Supplementary Fig. 3 for more details]. We are able to cancel magnetic gradients to better than a few $100 \mu \mathrm{G} / \mathrm{cm}$ along the weak trapping direction $x$. Residual magnetic forces along the $y$ - and $z$-directions are negligible due to the stronger confinement.

Spin-1 Gross-Pitaevskii equations. In the 1d limit, the complete BEC wavefunction can be written as $\boldsymbol{\Psi}=\phi_{\perp}(y, z) \zeta(x)$ where $\phi_{\perp}(y, z)$ denotes the transverse harmonic oscillator ground state. The one-dimensional spin-1 Gross-Pitaevskii equation can be written as a set of three equations for each Zeeman component, of the form

$$
\begin{aligned}
& i \hbar \frac{\partial \zeta_{+1}}{\partial t}=\left[\mathcal{L}+g_{s}^{1 \mathrm{~d}}\left(\rho_{1 \mathrm{~d}, 0}+m_{\|}\right)\right] \zeta_{+1}+g_{s}^{1 \mathrm{~d}} \zeta_{0}^{2} \zeta_{-1}^{*}, \\
& i \hbar \frac{\partial \zeta_{0}}{\partial t}=\left[\mathcal{L}+g_{s}^{1 \mathrm{~d}}\left(\rho_{1 \mathrm{~d}}-\rho_{1 \mathrm{~d}, 0}\right)\right] \zeta_{0}+2 g_{s}^{1 \mathrm{~d}} \zeta_{0}^{*} \zeta_{-1} \zeta_{+1}, \\
& i \hbar \frac{\partial \zeta_{-1}}{\partial t}=\left[\mathcal{L}+g_{s}^{1 \mathrm{~d}}\left(\rho_{1 \mathrm{~d}, 0}-m_{\|}\right)\right] \zeta_{-1}+g_{s}^{1 \mathrm{~d}} \zeta_{0}^{2} \zeta_{+1}^{*},
\end{aligned}
$$

with $\mathcal{L}=-\frac{\hbar^{2}}{2 M_{\mathrm{Na}}} \partial_{x}^{2}+\frac{1}{2} M_{\mathrm{Na}} \omega_{x}^{2} x^{2}+E_{\text {Zeeman }}+\bar{g}^{1 \mathrm{~d}} \rho_{1 \mathrm{~d}}$ the spin-independent GP operator and $m_{\|}=\rho_{1 \mathrm{~d},+1}-\rho_{1 \mathrm{~d},-1}$ the magnetization density.

We propagate Eq. (8) in imaginary time to obtain the lowest energy state using a split-step method. The evolution due to the kinetic energy, local spinindependent and local spin-dependent terms are calculated separately by exponentiating the corresponding operator. This can be done analytically, either in the momentum or position basis. Then the total evolution at each time step is approximated by multiplying all three evolution operators neglecting their commutation properties (first-order Trotter expansion). We have studied the influence of the time step carefully to make sure the higher-order terms are indeed negligible.

We use harmonic oscillator units where time is rescaled by $\omega_{x}^{-1}$, energy by $\hbar \omega_{x}$, and lengths by $a_{x}=\sqrt{\hbar /\left(m_{\mathrm{Na}} \omega_{x}\right)}$. For the data shown in this paper, we typically use a grid containing $N=64$ points and toal length $30_{a x}$, an imaginary time step $\omega_{x} \delta t=2 \times 10^{-5}$ and we compute the imaginary time evolution up to $\omega_{x} T=10^{3}$. We use dimensionless coupling constants $N \bar{g}^{1 \mathrm{~d}}=N \bar{g} /\left(2 \pi \hbar \omega_{x} a_{y}^{2} a_{x}\right) \approx 378.9$ and $g_{\mathrm{s}}^{1 \mathrm{~d}} / \bar{g}^{1 \mathrm{~d}}=0.0357$.

\section{Data availability}

The data that support the findings of this study and numerical programs used for data analysis are available from the corresponding author upon reasonable request.

Received: 23 July 2018 Accepted: 7 January 2019

Published online: 29 March 2019

\section{References}

1. Pitaevskii, L. \& Stringari, S. Bose Einstein Condensation, International Series of Monographs on Physics vol. 116 (Oxford University Press, Oxford, 2003).

2. Kawaguchi, Y. \& Ueda, M. Spinor Bose-Einstein condensates. Phys. Rep. 520 253-381 (2012).

3. Stamper-Kurn, D. M. \& Ueda, M. Spinor Bose gases: symmetries, magnetism and quantum dynamics. Rev. Mod. Phys. 85, 1191-1244 (2013).

4. Chang, M.-S. et al. Observation of spinor dynamics in optically trapped ${ }^{87} \mathrm{Rb}$ Bose-Einstein condensates. Phys. Rev. Lett. 92, 140403 (2004).

5. Schmaljohann, H. et al. Dynamics of $f=2$ spinor Bose-Einstein condensates. Phys. Rev. Lett. 92, 040402 (2004).

6. Kuwamoto, T., Araki, K., Eno, T. \& Hirano, T. Magnetic field dependence of the dynamics of ${ }^{87} \mathrm{Rb}$ spin-2 Bose-Einstein condensates. Phys. Rev. A 69, 063604 (2004)

7. Chang, M.-S., Qin, Q., Zhang, W., You, L. \& Chapman, M. S. Coherent spinor dynamics in a spin-1 Bose condensate. Nat. Phys. 1, 111-116 (2005)

8. Kronjäger, J., Becker, C., Navez, P., Bongs, K. \& Sengstock, K. Magnetically tuned spin dynamics resonance. Phys. Rev. Lett. 97, 110404 (2006).

9. Black, A. T., Gomez, E., Turner, L. D., Jung, S. \& Lett, P. D. Spinor dynamics in an antiferromagnetic spin-1 condensate. Phys. Rev. Lett. 99, 070403 (2007).

10. Pechkis, H. K. et al. Spinor dynamics in an antiferromagnetic spin-1 thermal Bose gas. Phys. Rev. Lett. 111, 025301 (2013).

11. Lahaye, T., Menotti, C., Santos, L., Lewenstein, M. \& Pfau, T. The physics of dipolar bosonic quantum gases. Rep. Progr. Phys. 72, 126401 (2009). 
12. Pasquiou, B. et al. Spontaneous demagnetization of a dipolar spinor Bose gas in an ultralow magnetic field. Phys. Rev. Lett. 106, 255303 (2011).

13. Fava, E. et al. Observation of spin superfluidity in a Bose gas mixture. Phys. Rev. Lett. 120, 170401 (2018)

14. Kim, J. H., Seo, S. W. \& Shin, Y. Critical spin superflow in a spinor Bose-Einstein condensate. Phys. Rev. Lett. 119, 185302 (2017).

15. Modugno, G. et al. Collapse of a degenerate Fermi gas. Science 297, 2240-2243 (2002).

16. Günter, K., Stöferle, T., Moritz, H., Köhl, M. \& Esslinger, T. Bose-Fermi mixtures in a three-dimensional optical lattice. Phys. Rev. Lett. 96, 180402 (2006).

17. Ospelkaus, C., Ospelkaus, S., Sengstock, K. \& Bongs, K. Interaction-driven dynamics of ${ }^{40} \mathrm{~K}-{ }^{87} \mathrm{Rb}$ fermion-boson gas mixtures in the large-particlenumber limit. Phys. Rev. Lett. 96, 020401 (2006).

18. Ferlaino, F. et al. Feshbach spectroscopy of a $\mathrm{K}-\mathrm{Rb}$ atomic mixture. Phys. Rev. A 73, 040702 (2006).

19. Thalhammer, G. et al. Double species Bose-Einstein condensate with tunable interspecies interactions. Phys. Rev. Lett. 100, 210402 (2008).

20. Papp, S. B., Pino, J. M. \& Wieman, C. E. Tunable miscibility in a dual-species Bose-Einstein condensate. Phys. Rev. Lett. 101, 040402 (2008).

21. McCarron, D. J., Cho, H. W., Jenkin, D. L., Köppinger, M. P. \& Cornish, S. L. Dual-species Bose-Einstein condensate of ${ }^{87} \mathrm{Rb}$ and ${ }^{133}$ Cs. Phys. Rev. A 84, 011603 (2011).

22. Wacker, L. et al. Tunable dual-species Bose-Einstein condensates of ${ }^{39} \mathrm{~K}$ and ${ }^{87}$ Rb. Phys. Rev. A 92, 053602 (2015).

23. DeSalvo, B. J., Patel, K., Johansen, J. \& Chin, C. Observation of a degenerate Fermi gas trapped by a Bose-Einstein condensate. Phys. Rev. Lett. 119, 233401 (2017).

24. Shin, Y., Zwierlein, M. W., Schunck, C. H., Schirotzek, A. \& Ketterle, W. Observation of phase separation in a strongly interacting imbalanced Fermi gas. Phys. Rev. Lett. 97, 030401 (2006).

25. Myatt, C. J., Burt, E. A., Ghrist, R. W., Cornell, E. A. \& Wieman, C. E. Production of two overlapping Bose-Einstein condensates by sympathetic cooling. Phys. Rev. Lett. 78, 586-589 (1997).

26. Hall, D. S., Matthews, M. R., Ensher, J. R., Wieman, C. E. \& Cornell, E. A. Dynamics of component separation in a binary mixture of Bose-Einstein condensates. Phys. Rev. Lett. 81, 1539-1542 (1998).

27. De, S. et al. Quenched binary Bose-Einstein condensates: spin-domain formation and coarsening. Phys. Rev. A 89, 033631 (2014).

28. Nicklas, E. et al. Observation of scaling in the dynamics of a strongly quenched quantum gas. Phys. Rev. Lett. 115, 245301 (2015).

29. Lee, K. L. et al. Phase separation and dynamics of two-component Bose-Einstein condensates. Phys. Rev. A 94, 013602 (2016).

30. Stenger, J. et al. Spin domains in ground-state Bose-Einstein condensates Nature 396, 345-348 (1998).

31. Isoshima, T., Machida, K. \& Ohmi, T. Spin-domain formation in spinor Bose-Einstein condensation. Phys. Rev. A. 60, 4857-4863 (1999).

32. Miesner, H.-J. et al. Observation of metastable states in spinor Bose-Einstein condensates. Phys. Rev. Lett. 82, 2228-2231 (1999).

33. Stamper-Kurn, D. M. et al. Quantum tunneling across spin domains in a Bose-Einstein condensate. Phys. Rev. Lett. 83, 661-665 (1999).

34. Kronjäger, J., Becker, C., Soltan-Panahi, P., Bongs, K. \& Sengstock, K. Spontaneous pattern formation in an antiferromagnetic quantum gas. Phys. Rev. Lett. 105, 090402 (2010)

35. Matuszewski, M., Alexander, T. J. \& Kivshar, Y. S. Spin-domain formation in antiferromagnetic condensates. Phys. Rev. A 78, 023632 (2008).

36. Matuszewski, M., Alexander, T. J. \& Kivshar, Y. S. Excited spin states and phase separation in spinor Bose-Einstein condensates. Phys. Rev. A 80, 023602 (2009).

37. Matuszewski, M. Ground states of trapped spin-1 condensates in magnetic field. Phys. Rev. A 82, 053630 (2010).

38. Sadler, L. E., Higbie, J. M., Leslie, S. R., Vengalattore, M. \& Stamper-Kurn, D. M. Spontaneous symmetry breaking in a quenched ferromagnetic spinor Bose-Einstein condensate. Nature 443, 312-315 (2006).

39. Mur-Petit, J. et al. Dynamics of $f=1{ }^{87} \mathrm{Rb}$ condensates at finite temperatures. Phys. Rev. A 73, 013629 (2006).

40. Saito, H., Kawaguchi, Y. \& Ueda, M. Topological defect formation in a quenched ferromagnetic Bose-Einstein condensates. Phys. Rev. A 75, 013621 (2007).

41. Mur-Petit, J. Spin dynamics and structure formation in a spin-1 condensate in a magnetic field. Phys. Rev. A 79, 063603 (2009).

42. Vengalattore, M., Guzman, J., Leslie, S. R., Serwane, F. \& Stamper-Kurn, D. M. Periodic spin textures in a degenerate $f=1{ }^{87} \mathrm{Rb}$ spinor Bose gas. Phys. Rev. A 81, 053612 (2010).

43. Guzman, J. et al. Long-time-scale dynamics of spin textures in a degenerate $f=1{ }^{87} \mathrm{Rb}$ spinor Bose gas. Phys. Rev. A 84, 063625 (2011).
44. Bookjans, E. M., Vinit, A. \& Raman, C. Quantum phase transition in an antiferromagnetic spinor Bose-Einstein condensate. Phys. Rev. Lett. 107, 195306 (2011).

45. Kang, S., Seo, S. W., Kim, J. H. \& Shin, Y. Emergence and scaling of spin turbulence in quenched antiferromagnetic spinor Bose-Einstein condensates. Phys. Rev. A. 95, 053638 (2017).

46. Swisłocki, T., Witkowska, E., Dziarmaga, J. \& Matuszewski, M. Double universality of a quantum phase transition in spinor condensates: Modification of the Kibble-Zurek mechanism by a conservation law. Phys. Rev. Lett. 110, 045303 (2013).

47. Petrov, D. S., Shlyapnikov, G. V. \& Walraven, J. T. M. Regimes of quantum degeneracy in trapped 1D gases. Phys. Rev. Lett. 85, 3745 (2000).

48. Petrov, D. S., Shlyapnikov, G. V. \& Walraven, J. T. M. Phase-fluctuating 3d Bose-Einstein condensates in elongated traps. Phys. Rev. Lett. 87, 050404 (2001).

49. Dettmer, S. et al. Observation of phase fluctuations in elongated Bose-Einstein condensates. Phys. Rev. Lett. 87, 160406 (2001).

50. Richard, S. et al. Momentum spectroscopy of 1d phase fluctuations in Bose-Einstein condensates. Phys. Rev. Lett. 91, 010405 (2003).

51. Hellweg, D. et al. Measurement of the spatial correlation function of phase fluctuating Bose-Einstein condensates. Phys. Rev. Lett. 91, 010406 (2003).

52. Hofferberth, S. et al. Probing quantum and thermal noise in an interacting many-body system. Nat. Phys. 4, 489-495 (2008).

53. Phuc, N. T., Kawaguchi, Y. \& Ueda, M. Effects of thermal and quantum fluctuations on the phase diagram of a spin- ${ }^{87} \mathrm{Rb}$ Bose-Einstein condensate. Phys. Rev. A 84, 043645 (2011).

54. Scherer, M. et al. Spontaneous breaking of spatial and spin symmetry in spinor condensates. Phys. Rev. Lett. 105, 135302 (2010).

55. Olf, R., Fang, F., Marti, G. E., MacRae, A. \& Stamper-Kurn, D. M. Thermometry and cooling of a Bose gas to 0.02 times the condensation temperature. Nat. Phys. 11, 720-723 (2015).

56. Roy, A. \& Angom, D. Thermal suppression of phase separation in condensate mixtures. Phys. Rev. A 92, 011601 (2015).

57. Frapolli, C. et al. Stepwise Bose-Einstein condensation in a spinor gas. Phys. Rev. Lett. 119, 050404 (2017).

58. Blakie, P. B., Bradley, A. S., Davis, M. J., Ballagh, R. J. \& Gardiner, C. W. Dynamics and statistical mechanics of ultra-cold Bose gases using $c$-field techniques. Adv. Phys. 57, 363-455 (2008).

59. Ho, T.-L. \& Zhou, Q. Obtaining the phase diagram and thermodynamic quantities of bulk systems from the densities of trapped gases. Nat. Phys. 6, 131-134 (2009).

60. Trebbia, J.-B., Esteve, J., Westbrook, C. I. \& Bouchoule, I. Experimental evidence for the breakdown of a Hartree-Fock approach in a weakly interacting Bose gas. Phys. Rev. Lett. 97, 250403 (2006).

61. Eto, Y. et al. Bouncing motion and penetration dynamics in multicomponent Bose-Einstein condensates. Phys. Rev. A 93, 033615 (2016).

62. Fradkin, E., Kivelson, S. A. \& Tranquada, J. M. Colloquium: theory of intertwined orders in high temperature superconductors. Rev. Mod. Phys. 87, 457-482 (2015).

63. Bienaimé, T. et al. Spin-dipole oscillation and polarizability of a binary Bose-Einstein condensate near the miscible-immiscible phase transition. Phys. Rev. A. 94, 063652 (2016).

64. Jacob, D. et al. Phase diagram of spin-1 antiferromagnetic Bose-Einstein condensates. Phys. Rev. A 86, 061601 (2012).

65. Zibold, T. et al. Spin-nematic order in antiferromagnetic spinor condensates. Phys. Rev. A 93, 023614 (2016).

66. Castin, Y. \& Dum, R. Bose-Einstein condensates in time-dependent traps. Phys. Rev. Lett. 77, 5315 (1996).

\section{Acknowledgments}

We acknowledge stimulating discussions with Emilia Witkowska, Sandro Stringari and Gabriele Ferrari. This work has been supported by ERC (Synergy Grant UQUAM) K.J.-G. acknowledges funding from the European Union's Horizon 2020 Research and Innovation Program under the Marie Sklodowska-Curie Grant Agreement No. 701894 LKB is a member of the SIRTEQ network of Région Ile-de-France.

\section{Author contributions}

K.J.-G. and A.I. contributed equally to this work. K.J.-G. and A.I. configured the existing experimental apparatus to perform 1D experiments. K.J.-G., A.I., and B.E. gathered the data with assistance from C.F. K.J.-G. and A.I. carried out the analysis of the data. K.J.-G., A.I, J.D., and F.G. performed numerical and analytical calculations. All authors contributed to discussions and preparation of the manuscript. This work was supervised by J.D. and F.G. 


\section{Additional information}

Supplementary Information accompanies this paper at https://doi.org/10.1038/s41467019-08505-6

Competing interests: The authors declare no competing interests.

Reprints and permission information is available online at http://npg.nature.com/ reprintsandpermissions/

Journal peer review information: Nature Communication thanks the anonymous reviewers for the contributions to the peer review of this work. Peer review reports are available.

Publisher's note: Springer Nature remains neutral with regard to jurisdictional claims in published maps and institutional affiliations. (c) (i) Open Access This article is licensed under a Creative Commons Attribution 4.0 International License, which permits use, sharing, adaptation, distribution and reproduction in any medium or format, as long as you give appropriate credit to the original author(s) and the source, provide a link to the Creative Commons license, and indicate if changes were made. The images or other third party material in this article are included in the article's Creative Commons license, unless indicated otherwise in a credit line to the material. If material is not included in the article's Creative Commons license and your intended use is not permitted by statutory regulation or exceeds the permitted use, you will need to obtain permission directly from the copyright holder. To view a copy of this license, visit http://creativecommons.org/ licenses/by/4.0/.

(C) The Author(s) 2019 